CAHIERS DE

NARRATOLOGIE

\section{Cahiers de Narratologie}

Analyse et théorie narratives

$24 \mid 2013$

Avant-gardes et littérature narrative

\title{
Les avant-gardes et la narration
}

Pour une poétique anti-prosaïque

\section{Tania Collani}

\section{(2) OpenEdition}

Journals

Édition électronique

URL : http://journals.openedition.org/narratologie/6661

DOI : 10.4000/narratologie.6661

ISSN : 1765-307X

Éditeur

LIRCES

Référence électronique

Tania Collani, «Les avant-gardes et la narration », Cahiers de Narratologie [En ligne], 24 | 2013, mis en ligne le 17 septembre 2013, consulté le 30 avril 2019. URL : http://journals.openedition.org/ narratologie/6661; DOI : 10.4000/narratologie.6661

Ce document a été généré automatiquement le 30 avril 2019

Article L.111-1 du Code de la propriété intellectuelle. 


\title{
Les avant-gardes et la narration
}

\author{
Pour une poétique anti-prosaïque
}

\author{
Tania Collani
}

1 La définition de roman est tellement vaste, aujourd'hui comme au début du XXe siècle, qu'il serait extrêmement réductif de prendre au pied de la lettre la répulsion manifestaire diffusée chez les avant-gardistes vis-à-vis du genre romanesque. Comme je l'ai écrit pour les surréalistes dans mon travail sur la prose surréaliste européenne, ils "partagent plutôt une certaine antipathie (d'ailleurs diffusée par l'esprit avant-gardiste contemporain) à l'égard du roman, en tant que pratique narrative à la base de la culture bourgeoise du siècle précédent ${ }^{1} »$. Au cours du XIX ${ }^{\mathrm{e}}$ siècle, le roman était devenu un phénomène populaire et quelque peu massifié, décliné dans une myriade de sous-genres très répandus dans cette première partie $\mathrm{du} \mathrm{XX}^{\mathrm{e}}$ siècle qui fait l'objet de ma réflexion (policier, fantastique, noir, d'aventures, psychologique, scientifique, etc.). Il représentait de ce fait le canon à déstructurer.

2 En défrichant davantage le terrain théorique autour du genre romanesque dans le contexte des avant-gardes, j'aimerais tout d'abord reprendre ce que Michel Dupuis écrit dans son étude sur le roman à cette époque :

Qui dit « récit » ou "roman» manipule implicitement trois concepts : celui de la narration (texte, techniques de composition), celui de fiction (le monde romanesque à l'intellection duquel la narration nous invite), celui enfin de réalité avec laquelle la fiction entretient certains rapports. $\mathrm{Au} \mathrm{XX}^{\mathrm{e}}$ siècle, le roman [...] poursuit fondamentalement un processus d'affranchissement vis-à-vis du récit traditionnel ${ }^{2}$.

3 Au fond, le mépris du roman au niveau des différents groupes d'avant-garde est motivé d'une part par le fait qu'il représentait la tradition; d'autre part, l'attitude profondément innovante des futuristes, dadaïstes et surréalistes menait naturellement à une exaltation de la dimension "poétique » au détriment du "prosaïsme ", entendu comme tout ce qui ressort du quotidien et de la banalités. Il s'agit d'une posture qui reprend la vision quelque peu élitiste et hermétique inaugurée par Mallarmé dans sa "Crise de Vers ", à la fin du XIXe siècle, dans laquelle il n'hésitait pas à prendre ses distances du prosaïsme de la narration, accusée d'étouffer la magie vibratoire du message : 
Narrer, enseigner, même décrire, cela va et encore qu'à chacun suffirait peut-être pour échanger la pensée humaine, de prendre ou de mettre dans la main d'autrui en silence une pièce de monnaie. [...] À quoi bon la merveille de transposer un fait de nature en sa presque disparition vibratoire selon le jeu de la parole, cependant ${ }^{4}$.

Plusieurs chercheurs se sont déjà interrogés sur l'existence d'une dimension narrative dans la production des avant-gardes européennes. Luciano De Maria, par exemple, se posait la question à propos de Marinetti et des futuristes :

Existe-t-il une littérature narrative futuriste? Une littérature narrative d'avantgarde, je veux dire, différente par rapport aux schémas romanciers de l'époque et caractérisée par des traits indélébilement propres. Je pense que Mafarka le futuriste et Les Indomptables de Marinetti, Le Code de Perelà de Palazzeschi, L'Ellipse et la Spirale de Buzzi et Sam Dunn est mort de Corra se distinguent de la littérature narrative italienne de ces années en raison d'une tendance à l'antiréalisme, à l'allégorisme et à l'ésotérisme qui, à de différents degrés, définissent les romans cités ${ }^{5}$.

La question du roman et des avant-gardes est effectivement à placer dans une perspective plus vaste de l'histoire littéraire - les futuristes, tout comme les surréalistes plus tard, écrivant des romans (ou mieux, des récits) stylistiquement originaux pour se différencier par rapport au canon de l'époque. Luciano De Maria met très bien en avant l'importance de certaines thématiques (comme l'exotérisme, et j'ajouterais la ville moderne, la rencontre, l'atmosphère onirique, le hasard, l'imprévu et l'automatisme) et de certains choix de poétique (comme l'antiréalisme et, j'ajouterais, l'ironie et la démystification, l'expérimentation langagière, l'analogie, l'hybridation stylistique), qui rendent cette production avant-gardiste facilement reconnaissable. L'attitude antiréaliste, la destruction de la grille spatio-temporelle figée qui captive et rassure le lecteur des romans naturalistes, sans oublier l'adoption des nouveaux préceptes en matière de poétique diffusés par les manifestes, mènent vers ces textes que Dominique Rabaté et Jean-Yves Tadié ont justement défini des " récits poétiques ${ }^{6} »:$ « Privilégiant le flou ou la demi-teinte, ce type de récit s'ouvre à une écriture plus ouvertement poétique, cherchant à structurer musicalement ses motifs, recourant abondamment aux prestiges de la métaphore ${ }^{7}$ ».

Luca D'Ascia va dans le même sens lorsqu'il écrit que le roman futuriste incarne la tentative de traduction, en langage narratif, des innovations techniques et stylistiques élaborées dans les champs de la poésie, des arts, de la politique et de la propagande. C'est dans ce contexte que le roman futuriste est fortement visuel, car il remplace la diachronie du roman traditionnel par une succession rapide d'images (et évidemment d'autres pistes s'ouvriraient vers l'imagisme d'Ezra Pound): «Le roman futuriste est un compromis entre la thématique fin-de-siècle de tradition symboliste et les principes esthétiques de l'avant-garde : simultanéité, visualité, théâtralisation, poly-expressivité ${ }^{8}$ ».

7 Dans son travail monumental sur Le Surréalisme et le roman, Jacqueline Chénieux prend quelques-unes des tensions fondantes du roman (vrai-fictif, crédible-vraisemblable, l'adhésion émotionnelle du lecteur, etc.) et elle ajoute à la vision du dépassement de la tradition une autre variante fondamentale pour saisir l'originalité de ce corpus de textes : « la narration romanesque requiert en fait de la part du lecteur une croyance qui n'est pas crédulité devant l'illusoire créé ${ }^{~}$. Ainsi, grâce à la médiation du merveilleux, les surréalistes (au moins ceux qui sont dans l'entourage proche d'André Breton) adoptent la modalité narrative, tout en brouillant les trois concepts de narration, fiction et réalité, justement dans le but de rendre vraisemblable ce qui ne l'est pas, de «rendre réel le possible, le rêve, l'inouï ${ }^{10}$ ". 
8 Si l'on s'arrête sur le foisonnement critique autour de la question, il n'y a plus aucun doute : la narration avant-gardiste, le "roman d'avant-garde ", a ses traits distinctifs. Michel Dupuis en repère un parmi les plus importants dans le dialogue collectif des différents mouvements et courants, et il repère deux notions essentielles : «D'abord le contact direct avec d'autres formes d'art, notamment la poésie et les arts plastiques. Ensuite le caractère généralement expérimental, quoique théorique, de la création romanesque ${ }^{11} »$. Barbara Meazzi, tout en constatant la difficulté de classement de la prose futuriste, souligne parmi les traits caractéristiques « le besoin de rajouter un sous-titre ou spécifier le type de $\operatorname{roman}^{12} "$, attitude d'ailleurs reprise ou partagée par certains surréalistes (Anicet ou le panorama, roman d'Aragon, ou Babylone, roman de René Crevel, par exemple). Et elle n'hésite pas à mettre en avant la façon selon laquelle la « cohérence de cette production surgit de plusieurs éléments: une écriture dans l'ensemble assez fragmentaire, caractéristique de l'époque [...] ce renouvellement se produit tantôt grâce à la commixtion des genres, tantôt grâce à l'incessante opposition et juxtaposition de synthèse et analyse, illusion et réalité, silence et parole, rationnel et a-logicité, réalisme et anti-réalisme ${ }^{13} »$.

Le jeu entre les frontières de la prose et de la poésie, du réel et de l'irréel, de l'impossible et pourtant vraisemblable, confère cette atmosphère exotérique et merveilleuse à certains romans avant-gardistes, en abattant les barrières définitoires entre les différents mouvements. Comment arrive-t-on à dire la poésie, la magie, l'expression de l'émotion vitale, en ayant recours à la forme du récit, sans pour autant tomber dans le prosaïsme?

\section{Anti-mimétisme. L'émotion contre la description}

10 Avant tout, pour écrire un roman avant-gardiste, il faut se libérer de la médiocrité mimétique inspirant bon nombre de romans : le fait de solliciter l'adhésion du lecteur ne comporte pas forcément le traitement de sujets communs en suivant la logique ordinaire, car la littérature et l'art en général représentent avant tout un moment de connaissance et d'expression pure, mis à la disposition de l'homme libre et pensant.

11 Dans son Manifeste Dada 1918, Tzara polémise avec l'attitude à la transparence de l'art qui tend à plaire et qui se rapproche dangereusement à la notion de journalisme : " L'art est une chose privée, l'artiste le fait pour lui ; une œuvre compréhensible est produit de journaliste ${ }^{14}$ ». Et plus loin il s'en prend aussi à la logique: "La logique est une complication. La logique est toujours fausse. Elle tire les fils des notions, paroles, dans leur extérieur formel, vers des bouts, des centres illusoires. Ses chaînes tuent, myriapode énorme asphyxiant l'indépendance ${ }^{15} »$. La transparence et la compréhension ne sont pas forcément deux caractères qui plaisent aux avant-gardistes; ils tendent donc à transposer la force expressive de la poésie (hermétisme, métaphores, onomatopées, etc.), dans la forme linéaire et raisonnante de la prose.

12 Le passage du Manifeste du surréalisme de 1924 contre les romans et le réalisme inspirés du positivisme est bien connu. André Breton attaque «l'attitude réaliste » accusée d'être « hostile à tout essor intellectuel et moral ${ }^{16}$ » et d'engendrer des « livres ridicules », en se fortifiant «sans cesse dans les journaux » et « en s'appliquant à flatter l'opinion dans ses goûts les plus bas ${ }^{17} »$. Tout comme Tzara, Breton critique la littérature-reportage (travail des journaux et des journalistes), « le style d'information pure et simple ${ }^{18}$ ", et n'oublie 
pas de prendre ses distances par rapport à «la clarté confinant à la sottise ${ }^{19}$ » et aux " petites observations » de certains romans.

"Je veux qu'on se taise, quand on cesse de ressentir ${ }^{20}$ "; en mettant en doute la fonction purement informative de l'art, André Breton n'hésite pas à déclencher une attaque frontale contre les descriptions, définies comme des "superpositions d'images de catalogue », où l'auteur recherche l'adhésion du lecteur avec des expédients faciles : « il cherche à me faire tomber d'accord avec lui sur des lieux communs ${ }^{21} »$. Les descriptions deviennent, dans cette perspective, des digressions à l'intérieur de la narration, des détours par des choses connues et évidentes, dont le résultat est le ralentissement de l'allure du récit.

Or, l'un des caractères de l'écriture d'avant-garde est justement sa volonté d'être synthétique, rapide, dense d'images. Après avoir affirmé sa haine pour la ligne courbe, les détours et les tourniquets, Marinetti n'hésite pas à postuler son « Amour pour la vitesse, pour l'abréviation et le résumé. "Raconte-moi tout, vite, en deux mots 22 !" ». Ce qui compte, c'est l'émotion, la mise en scène des effets d'un événement et non pas l'événement en soi ; dans ce sens nous pouvons comprendre l'exaltation du lyrisme vital, qui part certes du futurisme, mais qui touche aussi les autres avant-gardes :

L'impétuosité de la vapeur-émotion fera sauter le tuyau de la période, les soupapes de la ponctuation et les adjectifs que l'on dispose habituellement avec régularité comme des boulons. Vous aurez ainsi des poignées de mots essentiels se succédant en dehors de tout ordre conventionnel, votre ami n'ayant d'autre préoccupation que de rendre toutes les vibrations de son moi. [...] Ces rapports [que le poète et le public ont eus ensemble] ressemblent beaucoup à la camaraderie de deux vieux amis qui peuvent s'expliquer par un seul mot, un seul coup d'œil23.

Les mots se suivent de façon linéaire, comme dans la prose; mais leur choix et leur rapprochement très figuré se fait sur des critères poétiques et éminemment antiprosaïques. Et nous pouvons repérer cette tendance fréquemment dans la prose avantgardiste. Voici par exemple un extrait de Babylone (1927) de René Crevel, où est décrite, à travers les yeux de l'enfant protagoniste, la photo du père de la fillette, à côté d'une dénommée Cynthia, la jeune maîtresse pour laquelle il a abandonné le confort bourgeois :

L'enfant, prié d'aller chercher le quotidien anglais, dont la première page offre, entre la photographie d'un satyre de White Chapel quelques minutes avant sa pendaison, et une mariée médiévale à l'excès qui sort de Westminster au bras d'un jeune lourd impeccable et souriant, une Cynthia ruisselante de perles, si parfaite de cou et de visage, que, même en dépit de la triste matière du papier et de l'encre du journal, l'on croirait le front, les joues, les épaules, les bras polis par un soleil de bonheur, irisés d'un arc-en-ciel plus subtil que celui du triple sautoir sur la peau, la robe. Un poignet est si lourd de bracelets qu'une main, comme un oiseau mouillé au soleil d'avril, se repose sur la branche d'un fauteuil, tandis que l'autre, sèche de bagues, se rafraichît à l'écume des perles tombées en cascade du soyeux sommet des seins. L'écume de perles reçues en lac par le fragile plateau que fait de l'un à l'autre genou une robe de femme assise. Dans un petit rectangle qui découpe le miracle de la jupe, la tête du jeune père qui s'est laissé ensorceler ${ }^{24}$.

16 À propos des «prestiges de la métaphore »... La jeune et belle Cynthia devient, dans l'imaginaire de la petite fille, une sorte de Dame du lac, et ceci à travers un processus de suggestion plutôt que de description : les références à une "mariée médiévale ", à son être «ruisselante de perles »; les références aux champs sémantiques de l'eau et du lac (la main "comme un oiseau mouillé ", "l'écume des perles tombées en cascade", « l'écume de perles reçues en lac »); les références à la magie qui entoure sa personne (le 
satyre de White Chapel, les bracelets de gitane, les bras "polis par un soleil de bonheur " et « irisés d'un arc-en-ciel, le «miracle de la jupe » et son pouvoir ensorceleur - le jeune père "s'est laisse ensorceler »). Cet ensemble de références relève de la sphère d'une description évocatrice, d'une peinture anti-mimétique qui se focalise sur les images en éliminant le plus possible l'action - ce qui est témoigné par la rareté des verbes.

Au niveau formel, la construction des périodes est très inusitée : pour décrire le bras de la fille chargé de bracelets, le narrateur part du poignet, personnifiant la main qui accomplit l'acte de se reposer. De plus, afin de favoriser l'effet de perturbation, le poignet et la main de Cynthia deviennent, de manière générique, « un poignet » et " une main ». La poéticité de ce passage repose également sur des images nouvelles: "un oiseau mouillé au soleil d'avril et les «perles reçues en lac par le fragile plateau » illuminent la «triste matière du papier et de l'encre du journal ». Le « petit rectangle » qui encadre la tête du père à la fin de ce passage file la métaphore de la « tête » du père qui, dès le début de la citation, représente l'objet de la pendaison évoquée : la tête ensorcelée et pendue, qui gît dans le miracle du lac de la jupe, représente métaphoriquement les conséquences fatales de l'amour pour la fille aux cheveux de feu.

\section{L'image et l'analogie. Prose, c'est la vie}

Nous en arrivons à notre deuxième point : pour écrire un bon roman avant-gardiste, il faut user et abuser de l'analogie, figure de style par excellence de l'avant-garde, et incarnation de l'idée d'une utopique « imagination sans fils ». Il ne s'agit pas d'une pensée abstraite, métaphorique ou, moins encore, d'un processus de figuration symbolique ou symboliste, mais d'une démarche cognitive concrète, qui laisse entrevoir les fils fraîchement coupés reliant les mots et les choses. On ne part pas d'un mot pour en faire abstraction; on part de la réalité, toujours plurielle, rapide, en mouvement et, pour rendre cette réalité multi-facette, on rapproche brutalement les mots / choses.

Dans ses manifestes littéraires de 1912-1913, Marinetti est très clair à ce sujet: «L'analogie n'est que l'amour immense qui rattache les choses distantes, apparemment différentes et hostiles. C'est moyennant des analogies très vastes que ce style orchestral, à la fois polychrome, polyphonique et polymorphe, peut embrasser la vie de la matière ${ }^{25}$ ». L'hostilité des analogies futuristes n'est pas sans rappeler le degré d'arbitraire des images surréalistes: dans son Manifeste du surréalisme (1924), Breton essaye de proposer une classification d'images, et affirme que l'image « la plus forte est celle qui présente le degré d'arbitraire le plus élevé [...] celle qu'on met le plus longtemps à traduire en langage pratique $^{26} »$. Avant Breton, Reverdy avait publié son célèbre article sur l'image dans lequel il explicitait les bases de la poétique analogique : « L'image [...] ne peut naitre d'une comparaison mais du rapprochement de deux réalités plus ou moins éloignées. Plus les rapports des deux réalités rapprochées seront lointains et justes, plus l'image sera forte - plus elle aura de puissance émotive et de réalité poétique ${ }^{27}$ ».

Tzara aussi parcourt ce même chemin analogique et arbitraire, et dans son manifeste Dada manifeste sur l'amour faible et l'amour amer (1920), il recourt au signe mathématique " = " pour rapprocher des réalités qui ne partagent pas forcément de liens logiques: « $2=$ trois / canne = peut-être / après = déchiffrer [...] ou tout cela ensemble dans n'importe quel arrangement savoureux, savonneux, brusque ou définitif - tiré au sort est vivant ${ }^{28} »$. Par ailleurs, en parlant du Voleur de Talan (1917) de Reverdy, Tzara n'hésite 
pas à mettre en avant l'importance « cosmique » de cet ouvrage et des images qu'il livre à son lecteur : «Un livre inattendu, presque le roman qu'on a rêvé29 ». Plus loin, note-t-il encore: "Le roman de Reverdy est un poème. [...] Le Voleur de Talan est surtout un radiateur de vibration et les images qui se déchargent dans tous les sens (effet presque électrique à leur passage) se réunissent autour de lui ; l'œuvre de Reverdy est pour cela COSMIQUE ${ }^{30} »$.

Revenons un instant au futurisme et attardons-nous, à présent, sur la pensée de Marinetti ${ }^{31}$ : dans ses écrits théoriques, de manière significative Marinetti choisit des fragments de sa production en prose pour illustrer son style analogique. Au fond, la prose est censée raconter la vie ; si vraiment on veut changer la vie (une des grandes ambitions des avantgardes historiques), alors il faudra intervenir directement sur les modalités de dire et de penser le monde (le cosmos auquel se réfère Tzara) : « Pour envelopper et saisir tout ce qu'il y a de plus fuyant et de plus insaisissable dans la matière, il faut former des filets serrés d'images ou analogies qu'on lancera dans la mer mystérieuse des phénomènes ${ }^{32}$ ", écrit Marinetti dans son manifeste de 1912 Destruction de la syntaxe. Manifeste technique de la littérature futuriste. Et il n'a pas tort de choisir, parmi toutes les possibilités offertes par sa production, un fragment exemplaire de Mafarka le futuriste (1909-10), dans le chapitre intitulé "Les Forgerons de Milmillah »: au milieu de scènes pornographiques, d'exaltation de la force et du charisme de Mafarka, ce « roi tout-puissant qui gouverne sur toute l'Afrique ${ }^{33}$ ", entouré par des femmes qui s'offrent généreusement à lui, le héros se rend compte qu'il devra bientôt mourir, pour insuffler la vie à son enfant / automate, Gazourmah.

Son visage s'obscurcit... Il sentait son cœur rugir et sauter dans sa poitrine, comme un tigre, toutes ses griffes dehors !... Et chacune de ces griffes ouvrait dans sa chair des sillons parfumés, comme font les charrues dans la terre qu'excite le printemps.

La vie passée revenait vers lui, en poussant devant elle ses joies, détaillées et précises [...].

Toute l'âcre douceur de sa jeunesse montait de sa gorge, comme de la cour des écoles montent les cris des enfants vers leurs vieux maitres penchés aux parapets des terrasses d'où l'on voit fuir les bateaux sur la mer ${ }^{34}$.

Si j'ai choisi de compléter la citation de Marinetti en transcrivant le paragraphe suivant, c'est parce que je trouve que l'analogie filée sous-tendue au "filet serré d'images" arbitraires de la dernière phrase (l'oxymoron " âcre douceur "; la jeunesse et les cris des enfants présentent un même mouvement ascensionnel, mais ils ont inverti leur espace vital - les cris ne se situent plus dans la gorge, mais dans la cour, en laissant à la jeunesse qui «monte» cette sensation de suffocante douceur) ne peut être appréhendée qu'en comparant tous ces fragments de textes. L'idée du souvenir et du passé qui revient « en poussant » des images joyeuses, " détaillées et précises », dans un fond uniforme sillonné à plusieurs reprises (dans un premier moment c'est la chair ouverte par les griffes du félin; ensuite c'est la terre travaillée par les charrues, et à la fin ce sont les bateaux qui parcourent la mer), ne peut qu'être liée à ce moment d'introspection que vit Mafarka, dont les conséquences sont extrêmement charnelles et renvoient à son corps: son " visage s'obscurcit ", son " cœur rugit », ces sillons analogiques traversent sa chair, sa "gorge » est encombrée par « l'âcre douceur » de la jeunesse, ses yeux de maitre (car il n'est plus l'enfant) regardent vers cette mer qui n'est pas sans rappeler certains moments de la rêverie surréaliste. L'expédient qui vise à juxtaposer la dimension aquatique avec celle du rêve, du passé et de l'introspection n'est pas nouveau en littérature, et je ne citerai que la phrase de Rousseau, bien connue, contenue dans les Rêveries du promeneur 
solitaire: "Le flux et reflux de cette eau, son bruit continu mais renfle par intervalles frappant sans relâche mon oreille et mes yeux, suppléaient aux mouvements internes que la rêverie éteignait en moi et suffisaient pour me faire sentir avec plaisir mon existence, sans prendre la peine de penser ${ }^{35} »$.

Le choix de Marinetti de citer ce fragment dans son manifeste est à mon sens très éloquent : il semble vouloir diriger son lecteur vers une expérimentation analogique tout à fait surprenante pour sa profondeur auto-analytique, presque à vouloir donner une autre piste pour déchiffrer sa position souvent tranchante, vitaliste et destructrice. Il ne trahit pas celle qui sera sa position dans le manifeste de 1913, où il affirme que "ce conteur doué de lyrisme [...] lancera d'immenses filets d'analogies sur le monde, donnant ainsi le fond analogique et essentiel de la vie télégraphiquement ${ }^{36} »$. De la même manière, la prose douée de lyrisme mérite de raconter une vie.

\section{Hybridation : le lyrisme contre le récit}

Pour faire un vrai roman avant-gardiste, nous en arrivons à notre troisième partie, il faut le déstructurer génériquement, en se détachant le plus possible des formes du passé. De ce point de vue, la lecture que Michel Dupuis propose paraît très convaincante ; à son avis, les romanciers d'avant-garde se sont pris à la destruction de la «machine romanesque » de deux manières :

Certains [...] ont choisi, pour actualiser la rupture, le mode ironique. Le pastiche, la faille habilement introduite dans une architecture apparemment solide, sont pour eux les outils d'une vaste entreprise de dérision, d'un déboulonnage comique du passé. D'autres [...] ont travaillé à une mise en déroute réfléchie et opérante de l'attitude traditionnelle de lecture: dissection minutieuse qui, pour être le fruit d'une activité souvent hautement cérébrale [...] ouvrira d'autant mieux la voie à un renouveau de la relation écriture-lecture ${ }^{37}$.

Et si l'on parcourt le corpus des proses d'avant-garde, force est de constater qu'il y a effectivement des thématiques qui reviennent avec une certaine régularité, très probablement en raison de l'influence que le cinéma exerçait au niveau de l'imaginaire collectif : des héros solitaires qui sillonnent les villes modernes (à la manière de Fantômas ), les passages de détournement de scènes macabres (par exemple, des funérailles qui deviennent le prétexte pour susciter l'hilarité générale comme dans Entr'acte). Tout concourt à la remise en question de celle que Jacqueline Chénieux envisage comme une lecture " en acte ${ }^{38}$ ", en faisant référence à la sollicitation constante du lecteur, qui se doit d'être toujours actif dans sa lecture, sans pouvoir se permettre le répit offert par l'une de ces descriptions qu'on peut lire à lignes alternées.

Étant donné que la présente étude se focalise surtout sur des questions de poétique, je voudrais faire une incursion rapide dans la notion du «lyrisme", qui tend à mettre toujours en échec la modalité prosaïque du roman, en se manifestant dans les résultats stylistiques décrits plus haut. En 1913, Ardengo Soffici revient au moins à deux reprises sur la question du roman, à la fois pour mettre en avant sa nature négativement hybride et le besoin de le dépasser et pour le mélanger avec ce qu'il définit le « lyrisme pur » : « Le roman, la nouvelle, le drame sont des formes d'art hybrides, transitoires, destinées à disparaitre pour laisser le champ libre au lyrisme pur. - Et à l'autobiographie ${ }^{39}$ ». Dans le "Giornale di bordo » qu'il tient dans la revue Lacerba, Soffici se limite à une série de notes portant sur toutes sortes de sujets; sans vouloir forcément trouver une cohérence à ce 
qui ne l'a pas au départ, il me paraît tout de même significatif que, quelques mois plus tard, Soffici revienne sur la question pour rebondir, en laissant transparaitre ce qui était un sujet de réflexion commun aux futuristes. Pour écrire un bon roman futuriste, Soffici suggère que l'on parte de la conscience de l'échec de la forme romanesque en elle-même, accusée à plusieurs reprises d'être soumise aux thèses et à la rhétorique, qui relèguent la poésie et l'émotion à un plan tout à fait secondaire :

Si j'en avais le temps, je voudrais démontrer la nécessité d'un prochain échec du roman, de la nouvelle et du théâtre. [...] Des faits divers amalgamés à la poésie qui, pour le fait d'être subordonnée à la qualité de l'intrigue, présente forcément une qualité impure. [...] Des fragments de sensations directes tenus ensembles par un ciment en lest. Thèses et rhétoriques obligatoires. - Littérature agréable, utile à occuper du temps ${ }^{40}$.

Qu'est-ce ce lyrisme auquel font référence bon nombre de futuristes, dadaïstes et surréalistes, en l'opposant presque automatiquement à la linéarité de la prose? Il s'agit sûrement d'un condensé d'émotions subjectives devant détruire toute tendance à l'objectivation, toute perception du monde donnée ou imposée par la force des choses, et jamais remise en doute. Encore une fois, le problème de poétique déborde le champ strictement littéraire pour toucher des questions vitales. Par exemple, dans sa « Sensibilité futuriste et l'imagination sans fils » (1913), Marinetti déclare que « le lyrisme est la faculté très rare de se griser de la vie et de la griser de nous-mêmes; la faculté de transformer en vin l'eau trouble de la vie qui nous enveloppe et nous traverse ; la faculté de colorer le monde avec les couleurs spéciales de notre moi changeant ${ }^{41}$ ». Dans sa préface aux Locomotives avec des chaussettes (Le locomotive con le calze, 1919) d'Arnaldo Ginna, Bruno Corra définit « compositions ${ }^{42}$ » le genre de la prose pratiqué par son frère, et n'oublie pas d'en souligner « l'expression d'une subjectivité, qui n'est préoccupée que de se donner pour ce qu'elle est, en dépassant tous les cadres préétablis des genres littéraires du passé $e^{43}$ ».

28 Il y a une sorte de remise en doute collective concernant la structure monolithique d'un "sujet absolu » : le lyrisme est un concept très volubile au sein de l'avant-garde, il est lié aux différentes subjectivités, il n'est pas forcément ancré à la poésie et il exprime une posture émotionnelle bien marquée. En creusant dans la notion du lyrisme chez André Breton, Michel Murat souligne un emploi assez étrange de ce concept :

[...] le lyrisme est ce qui échappe «spasmodiquement» au contrôle. Or ce phénomène, qui devrait concerner les seuls énoncés lyriques (la poésie elle-même), affecte aussi l'usage que Breton fait de la notion dans des moments d'argumentation rationnelle: à plusieurs reprises le concept assez bizarrement échappe et devient retors - avant qu'une définition tardive ne le ressaisisse ${ }^{44}$.

L'exaltation de cette notion de "lyrisme", aux contours à la fois bien définis et très larges, présente le côté paradoxal qui caractérise la modernité définie par Compagnon: on prône la subjectivité au même moment où l'on rédige des manifestes programmatiques censés réunir un groupe autour d'un certain nombre de préceptes. La contradiction n'échappe pas à Tristan Tzara qui, dans son Manifeste dada 1918, écrit : «Je parle toujours de moi puisque je ne veux convaincre, je n'ai pas le droit d'entraîner d'autres dans mon fleuve, je n'oblige personne à me suivre et tout le monde fait son art à sa façon ${ }^{45}$ ».

Dans une attitude dialectique quelque peu tournée vers soi-même, Tzara expose prétentieusement son subjectivisme sans frontières dans un texte manifestaire; Soffici, quant à lui, met en avant l'importance de l'émotion, de l'autobiographie et du « lyrisme 
pur ", tandis que Marinetti et Breton laissent que le lyrisme se répande sur le monde, en une joyeuse contamination de formes et couleurs : autant de signaux qui rendent compte de la tentative de ramener le récit littéraire vers l'homme, à l'abri de toute notion de nature abstraite et absolue.

À mon sens, c'est justement cette poétique anti-prosaïque qui constitue la vraie clé de lecture sous-tendue aux productions narratives avant-gardistes, se traduisant dans des résultats formels récurrents: triomphe des analogies, hybridation générique, posture anti-descriptive, ironie, théorisation, pastiche. En restant toujours dans la perspective de l'anti-prosaïsme, la confrontation active que les avant-gardes entretiennent avec le genre narratif prend un sens plus profond: si l'on veut innover les différentes possibilités expressives, il faut commencer par la mise en échec des formes existantes. Certes, les conséquences s'en prennent notamment à la narration, à l'intrigue, au fil rouge qui parcourt les pages des romans "traditionnels", en laissant la place aux digressions poétiques, aux incursions génériques les plus disparates (théâtre, poésie, dessins, photos, etc.). Mais ce qui reste est surprenant dans sa tentative de conjonction des formes et des contenus : autant de joyaux littéraires qui ne cessent d'étonner les lecteurs.

\section{BIBLIOGRAPHIE}

Bernard, Suzanne, Le Poème en prose. De Baudelaire jusqu'à nos jours, Paris, Nizet, 1959.

Breton, André, Manifestes du surréalisme, Paris, Gallimard, 1985.

Chénieux, Jacqueline, Le Surréalisme et le roman, Lausanne, L'Âge d'Homme, « Lettera », 1983.

Collani, Tania, Le Merveilleux dans la prose surréaliste européenne, Paris, Hermann, 2010.

Crevel, René, Babylone : roman [1927], Paris, Pauvert, 1975.

De Maria, Luciano (éd.), Marinetti e i futuristi, Milan, Garzanti, 1994.

Dupuis, Michel, «Le roman », in Jean Weisgerber (dir.), Les Avant-gardes littéraires au XX ${ }^{e}$ siecle, vol. 2 : Théorie, Amsterdam, J. Benjamins, 1986.

Mallarmé, Stéphane, Igitur ; Divagations ; Un coup de dés, Paris, Gallimard, « Nrf », 2003.

Marinetti, Filippo Tommaso, « Les mots en liberté futuristes », Inter : art actuel, n 103, 2009, p. 18-25.

Marinetti, Filippo Tommaso, Tuons le clair de lune !! : Manifestes futuristes et autres proclamations, Paris, Fayard/Mille et une nuits, 2005.

Masi, Alessandro, Zig zag : il romanzo futurista [1995], Milan, Il Saggiatore, 2009.

Meazzi, Barbara, « Le Futurisme et le paradoxe réaliste : locomotives et chaussettes, ou du réalisme futuriste ", in Hubert Roland et al. (dir.), Les Frontières du réalisme dans la littérature narrative du XX $X^{e}$ siècle - The Borders of Realism in 20th Century Narrative Literature, 2004. p. 47-58. 
Murat, Michel, « "L'homme qui ment” : réflexions sur la notion de lyrisme chez Breton », in Dominique Rabaté, Joëlle de Sermet et Yves Vadé (dir.), Modernités. Le sujet lyrique en question, Talence, Presses Universitaires de Bordeaux, 1996, p. 151-164.

Rabaté, Dominique, Le Roman français depuis 1900, Paris, Puf, 1998.

Reverdy Pierre, «L'image » [1918], in Nord-Sud, Self defence et autres écrits sur l'art et la poésie, Paris, Flammarion, 1975, p. 73-75.

Rousseau, Jean-Jacques, Les Rêveries du promeneur solitaire [1782], in Euvres complètes, Paris, Gallimard, « Pléiade », 1959.

Soffici, Ardengo, « Giornale di bordo », Lacerba, n 3, 1er février 1913.

Tadié, Jean-Yves, Le Récit poétique, Paris, Gallimard, 1978.

Tzara, Tristan, Dada est tatou, tout est dada, Paris, Flammarion, 1996.

\section{NOTES}

1. Tania Collani, Le Merveilleux dans la prose surréaliste européenne, Paris, Hermann, 2010, p. 273.

2. Michel Dupuis, «Le roman », in Jean Weisgerber (dir.), Les Avant-gardes littéraires au XXe siècle, vol. 2 : Théorie, Amsterdam, J. Benjamins, 1986, p. 850.

3. À propos de la définition du « poème en prose », par exemple, Suzanne Bernard écrit : " Union insolite, sans doute, et impliquant une association de contraires (dans la langue courante, le “prosaïque" n'est-il pas le contraire du “poétique” ?) ». Cf. Suzanne Bernard, Le Poème en prose. De Baudelaire jusqu'à nos jours, Paris, Nizet, 1959, p. 434.

4. Stéphane Mallarmé, Igitur; Divagations; Un coup de dés, Paris, Gallimard, « Nrf », 2003, p. 251.

5. Nous traduisons (partout, sauf mention explicite). Luciano De Maria (éd.), Marinetti e i futuristi, Milan, Garzanti, 1994, p. XXVII : «Esiste una narrativa futurista? Una narrativa d'avanguardia, intendo, diversificata dagli schemi romanzeschi del tempo e accomunata da requisiti indelebilmente propri. Direi che Mafarka il futurista e Gli Indomabili di Marinetti, Il Codice di Perelà di Palazzeschi, L'Ellisse e la Spirale di Buzzi e Sam Dunn è morto di Corra si distinguano dalla narrativa italiana di quegli anni per una tendenza all'antirealismo, all'allegorismo e all'esoterismo che, in misura varia e diversa, definiscono i romanzi sopra nominati ».

6. Cf. Dominique Rabaté, Le Roman français depuis 1900, Paris, Puf, 1998 ; Jean-Yves Tadié, Le Récit poétique, Paris, Gallimard, 1978.

7. Dominique Rabaté, op. cit., p. 24-25.

8. Luca D'Ascia, «Il romanzo futurista: estetica dell'immagine e civiltà di massa », in Ilaria Ricconi (éd.), Arte d'avanguardia e società. L'esperienza futurista nel pensiero sociale e culturale contemporaneo, Rome, L'Albatros, 2006, p. 125 : «Il romanzo futurista è un compromesso fra la tematica fin de siècle di tradizione simbolista e i princìpi estetici dell'avanguardia : simultaneità, visività, teatralizzazione, poliespressività ».

9. Jacqueline Chénieux, Le Surréalisme et le roman, Lausanne, L'Âge d'Homme, 1983, p. 11.

10. Ibid., p. 17.

11. Michel Dupuis, «Le roman », loc. cit., p. 850

12. Barbara Meazzi, «Le Futurisme et le paradoxe réaliste : locomotives et chaussettes, ou du réalisme futuriste", in Hubert Roland et al. (dir.), Les Frontières du réalisme dans la littérature narrative du XXe siècle - The Borders of Realism in 20th Century Narrative Literature, 2004, p. 58.

13. Ibidem.

14. Tristan Tzara, Dada est tatou, tout est dada, Paris, Flammarion, 1996, p. 210.

15. Ibidem. 
16. André Breton, Manifestes du surréalisme, Paris, Gallimard, 1985, p. 16.

17. Ibidem.

18. Ibid., p. 17.

19. Ibid., p. 16.

20. Ibid., p. 18.

21. Ibid., p. 17.

22. Filippo Tommaso Marinetti, «L'immaginazione senza fili e le parole in libertà », Lacerba, $\mathrm{n}$

• 12, 15 juin 1913, p. 122 : « Amore per la velocità, dell'abbreviazione e del riassunto. "Raccontami tutto, presto, in due parole!"».

23. Filippo Tommaso Marinetti, «Les mots en liberté futuristes », Inter : art actuel, n 103, 2009, p. 21-22. En italien on apprécie davantage la condensation de certaines expressions et affirmations de ce manifeste publié pour la première fois le 11 mai 1913 : «L'irruenza del vapore-emozione farà saltare il tubo del periodo, le valvole della punteggiatura e i bulloni regolari dell'aggettivazione. Manate di parole essenziali senza alcun ordine convenzinale. Unica preoccupazione del narratore rendere tutte le vibrazioni del suo io. [...] Corrono infatti, fra il pubblico e il poeta, i rapporti stessi che esistono fra due vecchi amici. Questi possono spiegarsi con una mezza parola, un gesto, un'occhiata » (Filippo Tommaso Marinetti, «L'immaginazione senza fili e le parole in libertà ", Lacerba, n 12, 15 juin 1913, p. 122).

24. René Crevel, Babylone : roman [1927], Paris, Pauvert, 1975, p. 20-21.

25. Filippo Tommaso Marinetti, «Les mots en liberté futuristes », loc. cit., p. 22.

26. André Breton, op. cit., p. 50.

27. Pierre Reverdy, «L'image » [1918], in Nord-Sud, Self defence et autres écrits sur l'art et la poésie, Paris, Flammarion, 1975, p. 73.

28. Tristan Tzara, op. cit., p. 223.

29. Tristan Tzara, « Pierre Reverdy, Le Voleur de Talan », in ibid., p. 245.

30. Ibid., p. 246.

31. Pour une étude plus approfondie sur cet aspect appliqué à la prose surréaliste, je renvoie à mon volume.

32. Filippo Tommaso Marinetti, Tuons le clair de lune !!: Manifestes futuristes et autres proclamations, Paris, Fayard/Mille et une nuits, 2005, p. 50. En gras dans le texte.

33. Filippo Tommaso Marinetti, Mafarka le futuriste, Paris, Sansot, 1909, p. 229.

34. Ibid., p. 232.

35. Jean-Jacques Rousseau, Les Rêveries du promeneur solitaire [1782], in Euvres complètes, Paris, Gallimard, «Pléiade », 1959, p. 1045.

36. Filippo Tommaso Marinetti, « Les mots en liberté futuristes », loc. cit., p. 21-22.

37. Michel Dupuis, loc. cit., p. 850-851.

38. Jacqueline Chénieux-Gendron, op. cit., p. 34.

39. Ardengo Soffici, «Giornale di bordo », Lacerba, n 3, 1er février 1913, p. 27 : « Il romanzo, la novella, il dramma sono forme d'arte ibride, transitorie, destinate a sparire per lasciar libero il campo al puro lirismo. - E all'autobiografia ».

40. Ardengo Soffici, «Giornale di bordo », Lacerba, n 16, 15 août 1913, p. 183 : «Se ne avessi il tempo vorrei dimostrare la necessità del prossimo fallimento del romanzo, della novella e del teatro. [...] Fatti diversi rimpolpettati di poesia, la quale per essere subordinata alla necessità dell'intrigo è necessariamente di qualità impura. [...] Frammenti di sensazioni dirette tenuti insieme da un cemento di zavorra. Tesi et rettorica obbligatorie. - Letteratura amena, fatta per passare il tempo ».

41. Filippo Tommaso Marinetti, «Les mots en liberté futuristes », loc. cit., p. 21.

42. Dans l'anthologie d'Alessandro Masi, Zig zag: il romanzo futurista [1995], Milan, Il Saggiatore, 2009 , p. 173 : « composizioni ». 
43. Ibidem: «[...] sono l'espressione di una soggettività, non preoccupata d'altro che di dare se stessa, scavalcante tutti i quadri prestabiliti dei generi letterari passati ».

44. Michel Murat, " "L'homme qui ment" : réflexions sur la notion de lyrisme chez Breton », in Dominique Rabaté, Joëlle de Sermet et Yves Vadé (dir.), Modernités. Le sujet lyrique en question, Talence, Presses Universitaires de Bordeaux, 1996, p. 152.

45. Tristan Tzara, op. cit., p. 205.

\section{RÉSUMÉS}

Les avant-gardes européennes de la première moitié du XX $\mathrm{XX}^{\mathrm{e}}$ siècle tiennent un discours et une production étrangement (et souvent involontairement) unitaires vis-à-vis du roman : au niveau théorique, les futuristes, les dadaïstes et les surréalistes sont résolument contre cette forme littéraire qui incarne, bon gré mal gré, l'expression de l'esprit bourgeois du XIX ${ }^{\mathrm{e}}$ siècle ; toutefois, d'un point de vue pratique, force est de constater que ces mêmes avant-gardistes ont écrit plusieurs romans. Il ne s'agit pas de l'énième paradoxe caractérisant la modernité, mais plutôt d'une posture qui nécessite d'une réflexion autour de contraintes formelles inspirant la production prosaïque des avant-gardes et d'une analyse sur un corpus de textes en prose souvent laissés de côté en raison de leur hermétisme qui les rapproche davantage à la poésie.

The European Avant-gardes of the first half of the $20^{\text {th }}$ century held a strangely homogeneous (though often accidental) discourse on the novel: on a theoretical level, the Futurists, the Dadaists and the Surrealists were resolutely against a literary form which embodied the bourgeois spirit of the $19^{\text {th }}$ century. Nonetheless, these same avant-garde authors wrote several novels. This is not only another paradox of modernity, but rather a stance on the part of such writers which needs to be investigated. The paper offers a view of the formal constraints which inspired avant-gardes' prosaic production, as well as an analysis of a corpus of texts in prose often neglected because of their "hermetism", which brings them nearer to poetry than to novel.

\section{INDEX}

Mots-clés : poétique, prosaïsme, avant-garde, futurisme, dadaïsme, surréalisme

Index géographique : Italie, France, Suisse

Index chronologique : 1909-1930

\section{AUTEUR}

\section{TANIA COLLANI}

ILLE - UHA

Maître de conférences, chercheur affilié à l'Institut de recherche en langues et littératures européenne, Directrice du département d'italien de l'Université de Haute-Alsace. Docteur de l'Université de Bologne, spécialiste de l'avant-garde littéraire européenne, de la traduction et de la critique littéraire italienne, on lui doit plusieurs essais et traductions sur ces thématiques. 
Dernières publications : « La Poésie en prose et la prose en poésie : une lecture de Robert Desnos » (La Poésie en prose au $20^{e}$ siècle, Paris, Gallimard, 2012); Le Merveilleux dans la prose surréaliste européenne, Paris, Hermann, 2010 ; numéro spécial de L'Étoile de mer (Cahiers Robert Desnos), Paris, Gallimard, 2010. Plusieurs volumes d'actes de colloque : en collaboration avec Peter Schnyder : Seuils et Rites. Littérature et Culture, Paris, Orizons, 2009 et Critique littéraire et Littérature européenne, Paris, Orizons, 2010. En collaboration avec Noëlle Cuny : Poétiques scientifiques dans les revues de la modernité européennes (1900-1940), Paris, Classiques Garnier, 2013. 